knowledge. Although some of these variations reflect ignorance of existing evidence, others reflect a lack of appropriate evidence. The NHS should develop mechanisms for logging and reviewing these "informed" uncertainties, building on the experience of the NHS health technology assessment programme. $^{6}$ Indeed, when considering the massive investment in NHS information systems, is it not a scandal that they are not yet helping clinicians and patients to record and address uncertainties within the context of usual clinical care?

A prerequisite for constructive debate about uncertainties about the effects of treatments is a greater willingness among professionals and the public to admit and discuss them, combined with the humility to acknowledge that good intentions alone have not protected patients from the unintended harmful effects of treatments. Explicit admission of uncertainty by clinicians can sometimes undermine patients' confidence $^{8}$; it may even sometimes reduce the therapeutic effectiveness of individual encounters between clinician and patient. ${ }^{9}$ Finding ways to engage patients in the discussion that is needed thus poses a great challenge ${ }^{10}$ Nevertheless, because clinicians share with patients the front lines where uncertainties are

1 Smith R, Chalmers I. Britain's gift: a "Medline" of synthesised evidence. BMJ 2001;323:1437-1438.

2 Ashcroft R. Giving medicine a fair trial. BMJ 2000;320:1686

3 Chalmers I. It's official: evaluative research must become part of routine care in the NHS. JR Soc Med 2000;93:555-6.

4 Segelov E, Tattersall MH, Coates AS. Redressing the balance-the ethics of not entering an eligible patient on a randomised clinical trial. Ann Oncol 1992;3:103-5.

5 Gillon R. The function of criticism. BMJ 1981;283:1633-9.

6 Woods K. Filling the evidence gaps: identifying topics for research in the NHS health technology assessment programme. Clinical Excellence (in press).

7 Williams JG, Cheung WY, Cohen DR, Hutchings HA, Longo MF, Russel IT. Can randomised trials rely on existing electronic data? A feasibility encountered in practice, patients must obviously help define how to deal with these quandaries. ${ }^{11}$

Surely, however, it is up to clinicians to initiate these discussions-with each other, with patients, with the general public, and with researchers-who should certainly be challenged to be more responsive to the needs of people working in and using health services. As one philosopher-clinician has said recently, "a cowed medical profession has conceded too much of the running in medico-ethical debates to others." ${ }^{2}$ We need an alliance of clinicians, patients, researchers, and managers to discuss how best to deal with well informed uncertainties about the effects of treatments.

\section{Iain Chalmers coordinator}

James Lind Initiative, Oxford OX2 7LG (ichalmers@jameslindlibrary.org)

IC is indebted to Richard Ashcroft, Angela Coulter, Glyn Elwyn, Paul Glasziou, Jos Kleijnen, Alessandro Liberati, Andy Oxman, Dave Sackett, Trevor Sheldon, Ray Tallis, Hazel Thornton, Jan Vandenbroucke, John Williams, and Kent Woods for helpful comments on an earlier draft of this article.

Funding: IC receives a part time salary funded by the Medical Research Council and the Department of Health.

Competing interests: None declared.

study to explore the value of routine data in health technology assessment. Health Technol Assess 2003;7(26).

8 Ogden J, Fuks K, Gardner M, Johnson S, McLean M, Martin P, Shah R. Doctors expressions of uncertainty and patient confidence. Patient Educ Couns 2002;48:171-6.

9 Di Blasi Z, Harkness E, Ernst E, Georgiou A, Kleijnen J. Influence of context effects on health outcomes: a systematic review. Lancet 2001;357:75762.

10 Elwyn G. Shared decision making: patient involvement in clinical practice [PhD thesis]. Nijmegen: University of Nijmegen, Netherlands, 2001.

11 Feenberg A. On being a human subject: interest and obligation in the experimental treatment of incurable disease. Philosophical Forum 1992;23:213-30.

12 Tallis R. Ethical issues in medicine: setting the agenda. College commentary. London: Royal College of Physicians, September/October 2003:21.

\title{
Absence of evidence is not evidence of absence
}

\author{
We need to report uncertain results and do it clearly
}

$\mathrm{T}$ The title of this editorial is not new. For example, it was used nearly a decade ago for an article in the BMJ's Statistics Notes series. ${ }^{1}$ Altman and Bland considered the dangers of misinterpreting differences that do not reach significance, criticising use of the term "negative" to describe studies that had not found statistically significant differences. Such studies may not have been large enough to exclude important differences. To leave the impression that they have proved that no effect or no difference exists is misleading.

As an example, a randomised trial of behavioural and specific sexually transmitted infection interventions for reducing transmission of $\mathrm{HIV}-1$ was published in the Lancet. ${ }^{2}$ The incidence rate ratios for the outcome of HIV-1 infection were 0.94 (95\% confidence interval 0.60 to 1.45 ) and 1.00 (0.63 to 1.58 ) for two intervention groups compared with control. In the abstract, the interpretation is: "The interventions we used were insufficient to reduce HIV-1 incidence ..." But, looking again at the confidence intervals, the results in both treatment arms are compatible with a wide range of effects, from a $40 \%$ reduction in incidence of HIV-1 to a $50 \%$ increase. So, to give a summary of the results that gives the impression that this study has shown that these interventions are not capable of reducing HIV-1 incidence is misleading. What might be the implications for people at risk of HIV-1 infection? It could be that an intervention that does in fact protect against infection is not widely used. It could also be that an intervention that actually harms people by increasing HIV-1 infection is viewed as an intervention which has "no effect." The truth of these situations can be established only by collecting more evidence, and statements implying that an intervention has no effect might actually discourage further studies by giving the impression that the question has been answered. 


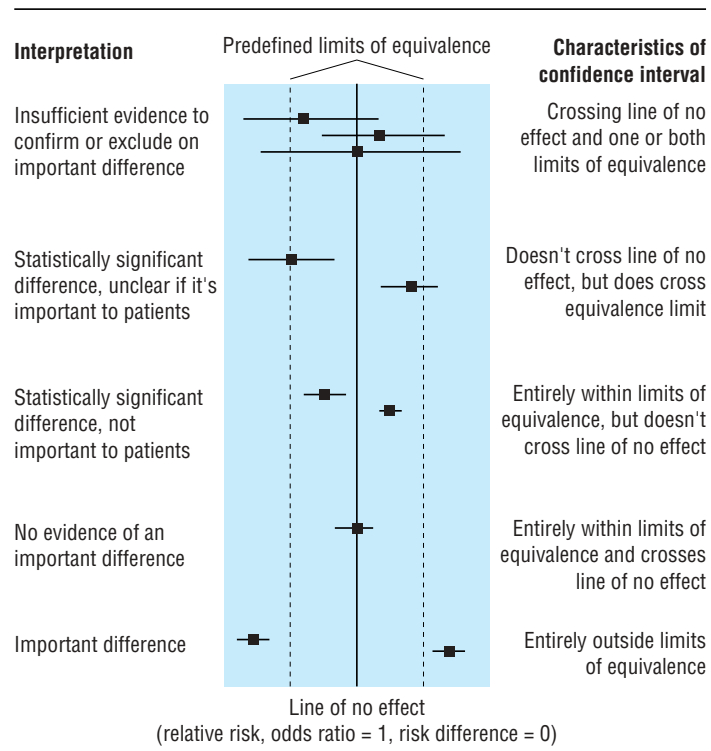

Relation between confidence interval, line of no effect, and thresholds for important differences (adapted from Armitage, Berry, and Matthews $\left.{ }^{4}\right)$

When is it reasonable to claim that a study has proved that no effect or no difference exists? The correct answer is "never," because some uncertainty will always exist. However, we need to have some rules for deciding when we are fairly sure that we have excluded an important benefit or harm. This implies that some threshold must be decided, in advance, for what size of effect is clinically important in that situation. This concept is not new and is used in designing equivalence studies, which set out to show whether one intervention is as good as another. ${ }^{3}$ Thresholds, often called limits of equivalence, are set between which an effect is designated as being too small to be important. Outcomes of, for example, studies of effectiveness can then be related to these thresholds. This is shown in the figure, where the confidence interval from a study is interpreted in the context of predefined limits of equivalence.

Of course, setting such thresholds is not straightforward. How big a reduction in the incidence of HIV-1 infection is important? How large an increase in incidence is important? Who should decide? How different should the thresholds be for different groups of patients and different outcomes? These are difficult questions, and although we may not be able to find easy answers to them, we can at least be more explicit in reporting what we have found in our research. Wording such as "our results are compatible with a decrease of this much or an increase of this much" would be more informative.

What can we do to help ensure that in another decade we will be closer to heeding the advice of Altman and Bland? Firstly, considering results of a particular study in the context of all available research which considers the same question can increase statistical power, reduce uncertainty, and thus reduce the confusing reporting of underpowered studies. Such an approach might have clarified the implications of a recent study of passive smoking published in the $B M J .^{5}$ Secondly, researchers need to be precise in their interpretation and language and avoid the temptation to save words by reducing the summary of the study to such an extent that the correct meaning is lost. Thirdly, journals need to be willing to publish uncertain results and thus reduce the pressure on researchers to report their results as definitive. ${ }^{6}$ We need to create a culture that is comfortable with estimating and discussing uncertainty.

Phil Alderson associate director

UK Cochrane Centre, Oxford OX2 7LG (palderson@cochrane.co.uk)

I thank Iain Chalmers and Mike Clarke for comments on draft versions.

Competing interests: None declared.

1 Altman DG, Bland JM. Absence of evidence is not evidence of absence. BMJ 1995;311:485.

2 Kamali A, Quigley M, Nakiyingi J, Kinsman J, Kengeya-Kayondo J, Gopal $\mathrm{R}$, et al. Syndomic management of sexually-transmitted infections and behaviour change interventions on transmission of HIV-1 in rural Uganda: a community randomised trial. Lancet 2003;361:645-52.

3 Greene WL, Concato J, Feinstein AR. Claims of equivalence in medical Greene WL, Concato J, Feinstein AR. Claims of equivalence in medical
research: are they supported by the evidence? Ann Intern Med research: are they

4 Armitage P, Berry G, Matthews JNS. Statistical methods in medical research. 4th ed. Oxford: Blackwell Science, 2002

5 Enstrom JE, Kabat GC. Environmental tobacco smoke and tobacco related mortality in a prospective study of Californians, 1960-98. BMJ 2003;326:1057-60.

6 Alderson P, Roberts I. Should journals publish systematic reviews that find no evidence to guide practice? Examples from injury research. $B M J$ 2000;320:376-7.

\section{Aspirin resistance}

\section{May be a cause of recurrent ischaemic vascular events in patients taking aspirin}

$\mathrm{A}$ spirin reduces the odds of serious atherothrombotic vascular events and death in a broad category of high risk patients by about one quarter. ${ }^{1}$ The primary antithrombotic mechanism is believed to be inhibition of the biosynthesis of thromboxane (and thus platelet activation) by inactivation of platelet cyclo-oxygenase- 1 . However, aspirin is not that effective. It still fails to prevent most (at least 75\%) serious vascular events in patients with symptomatic patients taking aspirin ("aspirin treatment failures") have many possible causes (box), and aspirin resistance has emerged as an additional contender. ${ }^{23}$

But what is aspirin resistance? Aspirin resistance has been used to describe several different phenomena. One is the inability of aspirin to protect patients from ischaemic vascular events. This has also been called clinical aspirin resistance. ${ }^{4}$ However, this definition is non-specific and could apply to any of the conditions listed in the box. Furthermore, it is not real- 